\title{
SHARP SOBOLEV INEQUALITIES ON THE COMPLEX SPHERE
}

\section{YAZHOU HAN AND SHUTAO ZHANG}

Abstract. This paper is devoted to establish a class of sharp Sobolev inequalities on the unit complex sphere as follows:

1) Case $0<d<Q=2 n+2$ : for any $f \in C^{\infty}$ and $2 \leqslant q \leqslant \frac{2 Q}{Q-d}$,

$$
\begin{aligned}
\|f\|_{q}^{2} \leqslant & \frac{8(q-2)}{d(Q-d)} \frac{\Gamma^{2}((Q-d) / 4+1)}{\Gamma^{2}((Q+d) / 4)}\left(\int_{\mathbb{S}^{2 n+1}} f \mathscr{A}_{d} f d \xi\right. \\
& \left.-\frac{\Gamma^{2}((Q+d) / 4)}{\Gamma^{2}((Q-d) / 4)} \int_{\mathbb{S}^{2 n+1}}|f|^{2} d \xi\right)+\int_{\mathbb{S}^{2 n+1}}|f|^{2} d \xi ;
\end{aligned}
$$

2) Case $d=Q$ : for any $f \in C^{\infty} \cap \mathbb{R} \mathscr{P}$ and $2 \leqslant q<+\infty$,

$$
\|f\|_{q}^{2} \leqslant \frac{q-2}{(n+1) !} \int_{\mathbb{S}^{2 n+1}} f \mathscr{A}_{Q}^{\prime} f d \xi+\int_{\mathbb{S}^{2 n+1}}|f|^{2} d \xi,
$$

where $\mathscr{A}_{d}(0<d<Q)$ are the intertwining operator, $\mathscr{A}_{Q}^{\prime}$ is the conditional intertwinor introduced in [2], and $d \xi$ is the normalized surface measure of $\mathbb{S}^{2 n+1}$.

Mathematics subject classification (2010): 26D10.

Keywords and phrases: Sharp Sobolev inequality, sharp Hardy-Littlewood-Sobolev inequality, complex sphere, CR manifold.

\section{REFERENCES}

[1] W. BeCKNER, Sharp Sobolev inequalities on the sphere and the Moser-Trudinger inequality, Annals of Mathematics, 138(1993), 213-242.

[2] T. P. BRANSON, L. FontANA AND C. MoRPuRgo, Moser-Trudinger and Beckner-Onofri's inequalities on the CR sphere, Annals of Mathematics, 177(2013), 1-52.

[3] S.-Y. A. Chang And P. YAng, Prescribing Gaussian curvature on $\mathbb{S}^{2}$, Acta Mathematica, 159(1987), 215-259.

[4] G. B. Folland, Spherical harmonic expansion of the Possion-Szegö kernel for the ball, Proc. Amer. Math. Soc., 47, 2(1975), 401-408.

[5] G. B. Folland AND E. M. STEIn, Estimates for the $\bar{\partial}_{b}$ complex and analysis on the Heisenberg group, Communications on Pure and applied Mathematics, 27(1994), 429-522.

[6] R. L. FRANK AND E. H. LiEB, Sharp constants in several inequalities on the Heisenberg group, Annals of Mathematics, 176(2012), 349-381.

[7] R. L. FrANK AND E. H. LIEB, A new, rearrangement-free proof of the sharp Hardy-LittlewoodSobolev inequality, Operator Theory: Advances and Applications, 219(2012), 55-67.

[8] M. Christ, H. LiU AND A. ZhANG, Sharp Hardy-Littlewood-Sobolev inequalities on the octonionic Heisenberg group, Calc. Var. PDE, 55, 11(2016),1-18.

[9] X. Han, G. LU AND J. ZhU, Hardy-Littlewood-Sobolev and Stein-Weiss inequalities and integral systems on the Heisenberg group, Nonlinear Anal. 75, 11 (2012), 4296-4314.

[10] D. Jerison And J. M. LeE, The Yamabe problem on CR manifolds, J. Differential Geom. 25 (1987): 167-197.

[11] D. JERISON AND J. M. LEe, Extremals for the Sobolev inequality on the Heisenberg group and the CR Yamabe problem, J. Amer. Math. Soc. 1 (1988): 1-13. 
[12] J. M. Lee And T. H. Parker, The Yamabe problem, Bull. Amer. Math. Soc. (N.S.), 17(1987): 3791.

[13] E. H. LiEB, Sharp constants in the Hardy-Littlewood-Sobolev and related inequalities, Ann. of Math. 118 (1983), 349-374.

[14] N. S. TRUDINGER, Remarks concerning the conformal deformation of Riemannian structures on compact manifolds, Annali Sc. Norm. supp. Pisa 22 (1968), 265-274.

[15] H. YAMABE, On the deformation of Riemannian structures on compact manifolds, Osaka Math. J., 12 (1960), 21-37. 